\title{
First and Second Fundamental Solutions of the Time-Fractional Telegraph Equation of Order $2 \alpha^{*}$
}

\author{
M. Ferreira ${ }^{\dagger, \ddagger} \quad$ M.M. Rodrigues ${ }^{\ddagger} \quad$ N. Vieira ${ }^{\ddagger}$ \\ $\dagger$ School of Technology and Management, \\ Polytechnic Institute of Leiria \\ P-2411-901, Leiria, Portugal. \\ ‡ CIDMA - Center for Research and Development in Mathematics and Applications \\ Department of Mathematics, University of Aveiro \\ Campus Universitário de Santiago, 3810-193 Aveiro, Portugal.
}

E-mails: milton.ferreira@ipleiria.pt, mrodrigues@ua.pt, nloureirovieira@gmail.com

\begin{abstract}
In this work we obtain the first and second fundamental solutions of the multidimensional time-fractional equation of order $2 \alpha, \alpha \in] 0,1]$, where the two time-fractional derivatives are in the Caputo sense. We obtain representations of the fundamental solutions in terms of Hankel transform, double Mellin-Barnes integral, and $\mathrm{H}$-functions of two variables. As an application, the fundamental solutions are used to solve a Cauchy problem, and to study telegraph process with Brownian time.
\end{abstract}

Keywords: Time-fractional telegraph equation; Telegraph Dirac operator; First and second fundamental solutions; Caputo fractional derivative; Multivariate Mittag-Leffler function; H-function of two variables.

MSC 2010: 30G35; 35R11, 26A33, 35A08, 35C15, 33E12.

\section{Introduction}

The telegraph equation is used as an alternative to the diffusion equation, since it has the potential to describe both diffusive and wave-like phenomena, due to the simultaneous presence of first and second order time derivatives. For example, in the case of the transport of energetic charged particle in turbulent magnetic fields such as low-energy cosmic rays in the solar wind, the diffusion equation can not be used to describe the transport for early times because it leads to a non-zero probability density everywhere, which would correspond to an infinite propagation speed. Using the telegraph equation in this case we get a more realistic model for the early phase transport because it combines diffusion with a finite propagation speed (see [18]). Telegraph equations have also an extraordinary importance in electrodynamics (the scalar Maxwell equations are of this type), in the theory of damped vibrations, and in probability because they are connected with finite velocity random motions (see $[12,16])$.

One of the first works studying the time-fractional telegraph equation is the paper of Cascaval et al. (see [4]). Here, the authors discussed some properties of the time-fractional telegraph equation in $\mathbb{R} \times \mathbb{R}^{+}$such as the well-posedness and the asymptotic behavior of the solutions, by using the Riemann-Liouville approach. In [15], Orsingher and Beghin obtained the fundamental solution of the time-fractional telegraph equation of order $2 \alpha$ in $\mathbb{R} \times \mathbb{R}^{+}$and gave a representation of their inverses in terms of stable densities. For the special case $\alpha=\frac{1}{2}$, the authors showed that the fundamental solution is the probability density of a telegraph process with Brownian

\footnotetext{
*The final version is published in AIP Conference Proceedings - ICNPAA 2018, 2046, (2018), Article No. 020079, 9 pp. It is available via the website: https://aip.scitation.org/doi/abs/10.1063/1.5081599
} 
time. In [3] it was discussed the solution of a general space-time fractional telegraph equation by means of the Laplace and Fourier transforms in the variables $x \in \mathbb{R}$ and $t \in \mathbb{R}^{+}$, respectively. In [19] it was obtained the solutions of the space-time fractional telegraph equation in $\mathbb{R} \times \mathbb{R}^{+}$in terms of Mittag-Leffler functions, using an operational approach. In [14], Mamchuev considered the inhomogeneous time-fractional telegraph equation with Caputo derivatives, and obtained a general representation of regular solution in rectangular domain in terms of fundamental solution and appropriate Green functions. Regarding the multidimensional case, in [5] the authors discussed and derived the solution of the time-fractional telegraph equation in $\mathbb{R}^{n} \times \mathbb{R}^{+}$with three kinds of nonhomogeneous boundary conditions, by the method of separation of variables. Very recently, in $[9,10]$ the authors found several representations of the fundamental solution of the time-fractional telegraph and telegraph Dirac equations in $\mathbb{R}^{n} \times \mathbb{R}^{+}$, in terms of integrals, special functions, and series.

The aim of this paper is to representations for the first and second fundamental solutions of the timefractional telegraph equation of order $2 \alpha$ in terms of Hankel transforms, double Mellin-Barnes integral and $\mathrm{H}$-functions of two variables. Moreover, the first fundamental solution is used in the law of telegraph process with Brownian time. This connection is motivated by the fact that the iterated Brownian motion and telegraph process with Brownian time are governed by time-fractional telegraph equations (see [15]).

The structure of the paper reads as follows: in the Preliminaries section we recall some basic facts about some special functions and fractional calculus, which are necessary for the development of the work. In the following section we obtain the first and second fundamental solutions of the time-fractional telegraph equation

$$
\left({ }^{C} \partial_{t}^{2 \alpha}+a^{C} \partial_{t}^{\alpha}-c^{2} \Delta_{x}\right) u(x, t)=0
$$

where $x \in \mathbb{R}^{n}, t>0,0<\alpha \leq 1, a \geq 0, c>0, \Delta_{x}$ is the Laplace operator in space, and the two time-fractional derivatives of orders $\alpha$ and $2 \alpha$ are in the Caputo sense, with $\alpha \in] 0,1]$. This section ends with an application to the resolution of a Cauchy problem. In the last section we present an application of our results to the law of a telegraph process with Brownian time.

\section{Preliminaries}

Here we recall the main tools concerning fractional derivatives and special functions that will be used in our work. We start by recalling the definition of the multivariate Mittag-Leffler function (see [13]).

Definition 2.The multivariate Mittag-Leffler function $E_{\left(a_{1}, \ldots, a_{n}\right), b}\left(z_{1}, \ldots, z_{n}\right)$ of n complex variables $z_{1}, \ldots, z_{n} \in$ $\mathbb{C}$ with complex parameters $a_{1}, \ldots, a_{n}, b \in \mathbb{C}$ (with positive real parts) is defined by

$$
E_{\left(a_{1}, \ldots, a_{n}\right), b}\left(z_{1}, \ldots, z_{n}\right)=\sum_{k=0}^{+\infty} \sum_{\substack{l_{1}+\ldots+l_{n}=k \\
l_{1}, \ldots, l_{n} \geq 0}}\left(\begin{array}{c}
k \\
l_{1}, \ldots, l_{n}
\end{array}\right) \frac{\prod_{i=1}^{n} z_{i}^{l_{i}}}{\Gamma\left(b+\sum_{i=1}^{n} a_{i} l_{i}\right)},
$$

where the multinomial coefficients are given by

$$
\left(\begin{array}{c}
k \\
l_{1}, \ldots, l_{n}
\end{array}\right):=\frac{k !}{l_{1} ! \times \ldots \times l_{n} !}
$$

When $n=2$ we obtain the bivariate Mittag-Leffler function, which can be written as

$$
E_{\left(a_{1}, a_{2}\right), b}\left(z_{1}, z_{2}\right)=\sum_{l_{1}=0}^{+\infty} \sum_{l_{2}=0}^{+\infty} \frac{\left(l_{1}+l_{2}\right) !}{l_{1} ! l_{2} !} \frac{z_{1}^{l_{1}} z_{2}^{l_{2}}}{\Gamma\left(b+a_{1} l_{1}+a_{2} l_{2}\right)}
$$

From (2) we can deduce, after straightforward calculations, an addition formula for the bivariate Mittag-Leffler function.

Lemma 2.2et $z_{1}, z_{2} \in \mathbb{C}$, and $a_{1}, a_{2}, b \in \mathbb{C}$ (with positive real parts). Then it holds

$$
E_{\left(a_{1}, a_{2}\right), b}\left(z_{1}, z_{2}\right)=\frac{1}{\Gamma(b)}+z_{1} E_{\left(a_{1}, a_{2}\right), b+a_{1}}\left(z_{1}, z_{2}\right)+z_{2} E_{\left(a_{1}, a_{2}\right), b+a_{2}}\left(z_{1}, z_{2}\right) .
$$


For general properties of the Mittag-Leffler function see $[11,13]$. Now we recall the definition of the H-function of two complex variables.

Definition 2.(3ee [1]) The H-function of two complex variables is defined via a double Mellin-Barnes integral of the form

$H_{p_{1}, n_{1} ; p_{2}, q_{2} ; p_{3}, q_{3}}^{0, n_{1} ; m_{2}, n_{2} ; m_{3}, n_{3}}\left[\begin{array}{c|c}x & \left(a_{j} ; \alpha_{j}, A_{j}\right)_{1, p_{1}} ;\left(c_{j}, \gamma_{j}\right)_{1, p_{2}} ;\left(e_{j}, E_{j}\right)_{1, p_{3}} \\ y & \left(b_{j} ; \beta_{j}, B_{j}\right)_{1, q_{1}} ;\left(d_{j}, \delta_{j}\right)_{1, q_{2}} ;\left(f_{j}, F_{j}\right)_{1, q_{3}}\end{array}\right]=\frac{1}{(2 \pi i)^{2}} \int_{\mathcal{L}_{2}} \int_{\mathcal{L}_{1}} \phi(s, w) \phi_{1}(s) \phi_{2}(w) x^{s} y^{w} d s d w$,

where

$$
\begin{aligned}
\phi(s, w) & =\frac{\prod_{i=1}^{n_{1}} \Gamma\left(1-a_{i}+\alpha_{i} s+A_{i} w\right)}{\prod_{i=n_{1}+1}^{p_{1}} \Gamma\left(a_{i}-\alpha_{i} s-A_{i} w\right) \prod_{j=1}^{q_{1}} \Gamma\left(1-b_{j}+\beta_{j} s+B_{j} w\right)}, \\
\phi_{1}(s) & =\frac{\prod_{j=1}^{m_{2}} \Gamma\left(d_{j}-\delta_{j} s\right) \prod_{i=1}^{n_{2}} \Gamma\left(1-c_{i}+\gamma_{i} s\right)}{\prod_{j=m_{2}+1}^{q_{2}} \Gamma\left(1-d_{j}+\delta_{j} s\right) \prod_{i=n_{2}+1}^{p_{2}} \Gamma\left(c_{i}-\gamma_{i} s\right)}, \\
\phi_{2}(w) & =\frac{\prod_{j=1}^{m_{3}} \Gamma\left(f_{j}-F_{j} w\right) \prod_{i=1}^{n_{3}} \Gamma\left(1-e_{i}+E_{i} w\right)}{\prod_{j=m_{3}+1}^{q_{3}} \Gamma\left(1-f_{j}+F_{j} w\right) \prod_{i=n_{3}+1}^{p_{3}} \Gamma\left(e_{i}-E_{i} w\right)},
\end{aligned}
$$

and where an empty product is interpreted as $1, x, y \in \mathbb{C}, m_{i}, n_{i}, p_{i}, q_{i} \in \mathbb{Z}$ such that $0 \leq m_{i} \leq q_{i}, 0 \leq n_{i} \leq p_{i}$ $(i=1,2,3) ; a_{i}, b_{j}, c_{i}, d_{j}, e_{i}, f_{j} \in \mathbb{C}, \alpha_{i}, A_{i}, \beta_{j}, B_{j}, \gamma_{i}, \delta_{j}, E_{i}, F_{j} \in \mathbb{R}^{+}$and the sequence of parameters $\left(a_{j}\right),\left(b_{j}\right)$, $\left(c_{j}\right),\left(d_{j}\right),\left(e_{j}\right)$ and $\left(f_{j}\right)$ are restricted that none of the poles of the integrand coincide. The contour $\mathcal{L}_{1}$ in the complex s-plane, and the contour $\mathcal{L}_{2}$ in the complex w-plane, are of Mellin-Barnes type with indentations, if necessary, to ensure that they separate one set of poles from the other.

In [1] the author proved that if

$$
\begin{aligned}
& \Omega_{1}=\sum_{i=1}^{n_{1}} \alpha_{i}-\sum_{i=n_{1}+1}^{p_{1}} \alpha_{i}-\sum_{i=1}^{q_{1}} \beta_{i}+\sum_{i=1}^{m_{2}} \delta_{i}-\sum_{j=m_{2}+1}^{q_{2}} \delta_{i}+\sum_{i=1}^{n_{2}} \gamma_{i}-\sum_{i=n_{2}+1}^{p_{2}} \gamma_{i} \geq 0 \\
& \Omega_{2}=\sum_{i=1}^{n_{1}} A_{i}-\sum_{i=n_{1}+1}^{p_{1}} A_{i}-\sum_{i=1}^{q_{1}} B_{i}+\sum_{i=1}^{m_{3}} F_{i}-\sum_{j=m_{3}+1}^{q_{3}} F_{i}+\sum_{i=1}^{n_{3}} E_{i}-\sum_{i=n_{3}+1}^{p_{3}} E_{i} \geq 0,
\end{aligned}
$$

and with the points $x=0$ and $y=0$ being tacitly excluded, the double Mellin-Barnes integral converges absolutely inside the sector given by

$$
|\arg (x)|<\frac{\pi}{2} \Omega_{1}, \quad|\arg (y)|<\frac{\pi}{2} \Omega_{2} .
$$

For $E_{\left(a_{1}, a_{2}\right), b}$ we have the following two results (see [9]).

Lemma 2.The bivariate Mittag-Leffler function $E_{\left(a_{1}, a_{2}\right), b}$ has the following representation in the form of double Mellin-Barnes integral

$$
E_{\left(a_{1}, a_{2}\right), b}\left(z_{1}, z_{2}\right)=\frac{1}{(2 \pi i)^{2}} \int_{\mathcal{L}_{1}} \int_{\mathcal{L}_{2}} \frac{\Gamma(1+s+w) \Gamma(-s) \Gamma(-w)}{\Gamma\left(b+a_{1} w+a_{2} s\right)} z_{2}^{s} z_{1}^{w} d w d s .
$$

Corollary 2.5he bivariate Mittag-Leffler function $E_{\left(a_{1}, a_{2}\right), b}$ has the following representation in the form of H-function of two variables

$$
E_{\left(a_{1}, a_{2}\right), b}\left(z_{1}, z_{2}\right)=H_{1,1 ; 0,1 ; 0,1}^{0,1 ; 1,0 ; 1,0}\left[\begin{array}{c|c}
z_{2} & (0 ; 1,1) ;-;- \\
z_{1} & \left(1-b ; a_{2}, a_{1}\right) ;(0,1) ;(0,1)
\end{array}\right] .
$$

In (5) and throughout the paper a horizontal line in the H-function means the absence of parameters. Now we recall the definition of the spaces $C_{\alpha}, \alpha \in \mathbb{R}$, and $C_{\alpha}^{m}, m \in \mathbb{N}$, given in [13]. 
Definition 2.(\$ee [13]) A real or complex-valued function $f(t), t>0$, is said to be in the space $C_{\alpha}, \alpha \in \mathbb{R}$, if there exists a real number $p>\alpha$ such that $f(t)=t^{p} f_{1}(t)$ for some function $f_{1} \in C[0, \infty)$.

It is easy to see that $C_{\alpha}$ is a vector space and the set of spaces $C_{\alpha}$ is ordered by inclusion according to $C_{\alpha} \subseteq C_{\beta} \Leftrightarrow \alpha \geq \beta$.

Definition 2.(Bee [13]) A function $f(t), t>0$, is said to be in the space $C_{\alpha}^{m}, m \in \mathbb{N}$, if and only if $f^{(m)} \in C_{\alpha}$.

Let $D_{t}^{\gamma}$ denotes the Caputo fractional derivative of order $\gamma>0$ defined by:

$$
D_{t}^{\gamma} u(t)=\left\{\begin{array}{cl}
\frac{1}{\Gamma(m-\gamma)} \int_{0}^{t}(t-s)^{-\gamma+m-1} u^{(m)}(s) d s, & m-1<\gamma<m \\
u^{(m)}(t), & \gamma=m
\end{array}\right.
$$

where $u^{(m)}:=\frac{d^{m} u}{d t^{m}}, m \in \mathbb{N}$ (see [2]). The next theorem will be used in our analysis and allow us to solve general linear fractional differential equations with constant coefficients and Caputo derivatives.

Theorem 2.(8ee [13, Thm 4.1]) Let $\mu>\mu_{1}>\ldots>\mu_{n} \geq 0, m_{i}-1<\mu_{i} \leq m_{i}, m_{i} \in \mathbb{N}, \lambda_{i} \in \mathbb{R}, i=1, \ldots, n$. Consider the initial value problem

$$
\left\{\begin{array}{l}
D_{t}^{\mu} y(t)-\sum_{i=1}^{n} \lambda_{i} D_{t}^{\mu_{i}} y(t)=g(t) \\
y^{(k)}(0)=c_{k} \in \mathbb{R}, \quad k=0, \ldots, m-1, \quad m-1<\mu \leq m,
\end{array}\right.
$$

where $g$ is assumed to lie in $C_{-1}$ if $\mu \in \mathbb{N}_{0}$ or in $C_{-1}^{1}$ if $\mu \notin \mathbb{N}_{0}$. Then (7) has a unique solution in the space $C_{-1}^{m}$ of the form

$$
y(t)=\int_{0}^{t} s^{\mu-1} E_{\left(\mu-\mu_{1}, \ldots, \mu-\mu_{n}\right), \mu}\left(\lambda_{1} s^{\mu-\mu_{1}}, \ldots, \lambda_{1} s^{\mu-\mu_{n}}\right) g(t-s) d s+\sum_{k=0}^{m-1} c_{k} u_{k}(t), \quad t \geq 0,
$$

with, $k=0, \ldots, m-1$

$$
u_{k}(t)=\frac{t^{k}}{k !}+\sum_{i=l_{k}+1}^{n} \lambda_{i} t^{k+\mu-\mu_{i}} E_{\left(\mu-\mu_{1}, \ldots, \mu-\mu_{n}\right), k+1+\mu-\mu_{i}}\left(\lambda_{1} t^{\mu-\mu_{1}}, \ldots, \lambda_{n} t^{\mu-\mu_{n}}\right) .
$$

The natural numbers $l_{k}, k=0, \ldots, m-1$ are determined from the conditions $m_{l_{k}} \geq k+1$ and $m_{l_{k}+1} \leq k$. In the case $m_{i} \leq k, i=0, \ldots, m-1$, we set $l_{k}:=0$, and of $m_{i} \geq k+1, i=0, \ldots, m-1$, then $l_{k}:=n$.

\section{First and second fundamental solution of the time-fractional tele- graph equation of order $2 \alpha$}

In this section we obtain the first and second fundamental solution of a particular case of the previous equation, where $\beta=2 \alpha$ and $0<\alpha \leq 1$, i.e., we look for a function $G^{\alpha}(x, t)$ that satisfies the following Cauchy problem

$$
\left\{\begin{array}{l}
\left({ }^{C} \partial_{t}^{2 \alpha}+a{ }^{C} \partial_{t}^{\alpha}-c^{2} \Delta_{x}\right) G^{\alpha}(x, t)=0 \\
G^{\alpha}(x, 0)=C_{0} \delta(x) \\
\frac{\partial G^{\alpha}}{\partial t}(x, 0)=C_{1} \delta(x)
\end{array}\right.
$$

where $x \in \mathbb{R}^{n}, t>0, a \geq 0, c>0, C_{0}, C_{1} \in \mathbb{R}, \delta(x)=\prod_{j=1}^{n} \delta\left(x_{j}\right)$ is the distributional Dirac delta function in $\mathbb{R}^{n}$. Applying the Fourier transform in $\mathbb{R}^{n}$ to the Cauchy problem we get the following initial-value problem

$$
\left\{\begin{array}{l}
\left({ }^{C} \partial_{t}^{2 \alpha}+a{ }^{C} \partial_{t}^{\alpha}+c^{2}|\kappa|^{2}\right) \widehat{G}^{\alpha}(\kappa, t)=0 \\
\widehat{G}^{\alpha}(\kappa, 0)=C_{0} \\
\frac{\partial \widehat{G}^{\alpha}}{\partial t}(\kappa, 0)=C_{1}
\end{array} .\right.
$$


To solve the problem (9), we apply Theorem 2.8 with $\lambda_{1}=-a, \lambda_{2}=-c^{2}|\kappa|^{2}, \mu=2 \alpha, \mu_{1}=\alpha, \mu_{2}=0, n=2$, $m=2, g(t)=0$, and $k=1$, obtaining the following solution

$$
\widehat{G}^{\alpha}(\kappa, t)=C_{0} \widehat{u}_{0}(\kappa, t)+C_{1} \widehat{u}_{1}(\kappa, t),
$$

where

$$
\begin{aligned}
& \widehat{u}_{0}(\kappa, t)=1-c^{2}|\kappa|^{2} t^{2 \alpha} E_{(\alpha, 2 \alpha), 1+2 \alpha}\left(-a t^{\alpha},-c^{2}|\kappa|^{2} t^{2 \alpha}\right), \\
& \widehat{u}_{1}(\kappa, t)=t-a t^{1+\alpha} E_{(\alpha, 2 \alpha), 2+\alpha}\left(-a t^{\alpha},-c^{2}|\kappa|^{2} t^{2 \alpha}\right)-c^{2}|\kappa|^{2} t^{1+2 \alpha} E_{(\alpha, 2 \alpha), 2+2 \alpha}\left(-a t^{\alpha},-c^{2}|\kappa|^{2} t^{2 \alpha}\right) .
\end{aligned}
$$

Taking into account Lemma 2.2 we have the following alternative representation of $\widehat{u}_{0}$ and $\widehat{u}_{1}$

$$
\begin{aligned}
& \widehat{u}_{0}(\kappa, t)=E_{(\alpha, 2 \alpha), 1}\left(-a t^{\alpha},-c^{2}|\kappa|^{2} t^{2 \alpha}\right)+a t^{\alpha} E_{(\alpha, 2 \alpha), 1+\alpha}\left(-a t^{\alpha},-c^{2}|\kappa|^{2} t^{2 \alpha}\right), \\
& \widehat{u}_{1}(\kappa, t)=t E_{(\alpha, 2 \alpha), 2}\left(-a t^{\alpha},-c^{2}|\kappa|^{2} t^{2 \alpha}\right) .
\end{aligned}
$$

From (2), we have the following representation of $\widehat{u}_{0}$ and $\widehat{u}_{1}$ in the form of a double series

$$
\begin{aligned}
\widehat{u}_{0}(\kappa, t)= & \sum_{p=0}^{+\infty} \sum_{q=0}^{+\infty} \frac{\Gamma(1+p+q)}{\Gamma(1+\alpha p+2 \alpha q) p ! q !}\left(-a t^{\alpha}\right)^{p}\left(-c^{2}|\kappa|^{2} t^{2 \alpha}\right)^{q} \\
& +a t^{\alpha} \sum_{p=0}^{+\infty} \sum_{q=0}^{+\infty} \frac{\Gamma(1+p+q)}{\Gamma(1+\alpha+\alpha p+2 \alpha q) p ! q !}\left(-a t^{\alpha}\right)^{p}\left(-c^{2}|\kappa|^{2} t^{2 \alpha}\right)^{q}, \\
\widehat{u}_{1}(\kappa, t)= & t \sum_{p=0}^{+\infty} \sum_{q=0}^{+\infty} \frac{\Gamma(1+p+q)}{\Gamma(2+\alpha p+2 \alpha q) p ! q !}\left(-a t^{\alpha}\right)^{p}\left(-c^{2}|\kappa|^{2} t^{2 \alpha}\right)^{q} .
\end{aligned}
$$

From Lemma 2.4 we have the following representations of $\widehat{u}_{0}$ and $\widehat{u}_{1}$ in the form of a double Mellin-Barnes integral

$$
\begin{aligned}
\widehat{u}_{0}(\kappa, t)= & \frac{1}{(2 \pi i)^{2}} \int_{\mathcal{L}_{1}} \int_{\mathcal{L}_{2}} \frac{\Gamma(1+s+w) \Gamma(-s) \Gamma(-w)}{\Gamma(1+\alpha w+2 \alpha s)}\left(a t^{\alpha}\right)^{w}\left(c^{2}|\kappa|^{2} t^{2 \alpha}\right)^{s} d w d s \\
& +\frac{a t^{\alpha}}{(2 \pi i)^{2}} \int_{\mathcal{L}_{1}} \int_{\mathcal{L}_{2}} \frac{\Gamma(1+s+w) \Gamma(-s) \Gamma(-w)}{\Gamma(1+\alpha+\alpha w+2 \alpha s)}\left(a t^{\alpha}\right)^{w}\left(c^{2}|\kappa|^{2} t^{2 \alpha}\right)^{s} d w d s, \\
\widehat{u}_{1}(\kappa, t)= & \frac{t}{(2 \pi i)^{2}} \int_{\mathcal{L}_{1}} \int_{\mathcal{L}_{2}} \frac{\Gamma(1+s+w) \Gamma(-s) \Gamma(-w)}{\Gamma(2+\alpha w+2 \alpha s)}\left(a t^{\alpha}\right)^{w}\left(c^{2}|\kappa|^{2} t^{2 \alpha}\right)^{s} d w d s .
\end{aligned}
$$

From Corollary 2.5 we can write $\widehat{u}_{0}$ and $\widehat{u}_{1}$ in the form of H-functions of two variables

$$
\begin{aligned}
& \widehat{u}_{0}(\kappa, t)=H_{1,1 ; 0,1 ; 0,1}^{0,1 ; 1,0 ; 1,0}\left[\begin{array}{c|c}
c^{2}|\kappa|^{2} t^{2 \alpha} & (0 ; 1,1) ;-;- \\
a t^{\alpha} & (0 ; 2 \alpha, \alpha) ;(0,1) ;(0,1)
\end{array}\right] \\
& +a t^{\alpha} H_{1,1 ; 0,1 ; 0,1}^{0,1 ; 1,0 ; 1,0}\left[\begin{array}{c|c}
c^{2}|\kappa|^{2} t^{2 \alpha} & (0 ; 1,1) ;-;- \\
a t^{\alpha} & (-\alpha ; 2 \alpha, \alpha) ;(0,1) ;(0,1)
\end{array}\right], \\
& \widehat{u}_{1}(\kappa, t)=t H_{1,1 ; 0,1 ; 0,1}^{0,1 ; 1,0 ; 1,0}\left[\begin{array}{c|c}
c^{2}|\kappa|^{2} t^{2 \alpha} & (0 ; 1,1) ;-;- \\
a t^{\alpha} & (-1 ; 2 \alpha, \alpha) ;(0,1) ;(0,1)
\end{array}\right] .
\end{aligned}
$$


Inverting the Fourier transform in (10) we get

$$
G^{\alpha}(x, t)=C_{0} u_{0}(x, t)+C_{1} u_{1}(x, t) .
$$

We will denote by $G_{1}^{\alpha}(x, t)=u_{0}(x, t)$ the first fundamental solution of the first equation of (8) that satisfies the initial conditions $G_{1}^{\alpha}(x, 0)=\delta(x)$ and $\frac{\partial G_{1}^{\alpha}}{\partial_{t}}(x, 0)=0$. Additionally, we denote by $G_{2}^{\alpha}(x, t)=u_{1}(x, t)$ the second fundamental solution of the first equation of (8) that satisfies the initial conditions $G_{2}^{\alpha}(x, 0)=0$ and $\frac{\partial G_{2}^{\alpha}}{\partial_{t}}(x, 0)=\delta(x)$. For obtaining the explicit expressions for $G_{1}^{\alpha}$ and $G_{2}^{\alpha}$ we recall the following formula (see [17])

$$
\frac{1}{(2 \pi)^{n}} \int_{\mathbb{R}^{n}} e^{-i \kappa \cdot x} \phi(|\kappa|) d \kappa=\frac{|x|^{1-\frac{n}{2}}}{(2 \pi)^{\frac{n}{2}}} \int_{0}^{+\infty} \phi(\tau) \tau^{\frac{n}{2}} J_{\frac{n}{2}-1}(\tau|x|) d \tau,
$$

where $J_{\nu}$ represents the Bessel function of first kind with index $\nu$, and the right hand side can be seen as a Hankel transform. We need also the following integral formula (see Formula (7) in [6, p. 22])

$$
\int_{0}^{+\infty} z^{\mu} J_{\nu}(z y)^{\frac{1}{2}} d z=\frac{2^{\mu+\frac{1}{2}}}{y^{\mu+1}} \frac{\Gamma\left(\frac{\mu}{2}+\frac{\nu}{2}+\frac{3}{4}\right)}{\Gamma\left(\frac{\nu}{2}-\frac{\mu}{2}+\frac{1}{4}\right)}
$$

which is valid under the condition $-\operatorname{Re}(\nu)-\frac{3}{2}<\operatorname{Re}(\mu)<-\frac{1}{2}$. Since the expressions for $G_{1}^{\alpha}$ are longer then the ones for $G_{2}^{\alpha}$, we are going to present only the calculations for $G_{2}^{\alpha}$. Applying the inverse Fourier transform to (12) and using (20), we get

$$
G_{2}^{\alpha}(x, t)=\frac{t|x|^{1-\frac{n}{2}}}{(2 \pi)^{\frac{n}{2}}} \int_{0}^{+\infty} \tau^{\frac{n}{2}} E_{(\alpha, 2 \alpha), 2}\left(-a t^{\alpha},-c^{2} \tau^{2} t^{2 \alpha}\right) J_{\frac{n}{2}-1}(\tau|x|) d \tau .
$$

Making use of Lemma 2.4, and interchanging the integrals due to the convergence, we obtain

$$
\begin{aligned}
G_{2}^{\alpha}(x, t)=\frac{t|x|^{1-\frac{n}{2}}}{(2 \pi)^{\frac{n}{2}}} \frac{1}{(2 \pi i)^{2}} \int_{\mathcal{L}_{1}} \int_{\mathcal{L}_{2}} \frac{\Gamma(1+s+w) \Gamma(-s) \Gamma(-w)}{\Gamma(2+\alpha w+2 \alpha s)}\left(a t^{\alpha}\right)^{w}\left(c^{2} t^{2 \alpha}\right)^{s} \\
\times \int_{0}^{+\infty} \tau^{\frac{n}{2}+2 s} J_{\frac{n}{2}-1}(\tau|x|) d \tau d w d s .
\end{aligned}
$$

Finally, using (21) and Corollary 2.5, we obtain, under the condition $-\frac{n}{2}<\Re(s)<-\frac{n}{4}$, the representation of the second fundamental solution in terms of double Mellin-Barnes integral and H-function of two variables:

$$
\begin{aligned}
& G_{2}^{\alpha}(x, t)=\frac{t}{\pi^{\frac{n}{2}}|x|^{n}} \frac{1}{(2 \pi i)^{2}} \int_{\mathcal{L}_{1}} \int_{\mathcal{L}_{2}} \frac{\Gamma(1+s+w) \Gamma\left(\frac{n}{2}+s\right) \Gamma(-w)}{\Gamma(2+\alpha w+2 \alpha s)}\left(a t^{\alpha}\right)^{w}\left(\frac{4 c^{2} t^{2 \alpha}}{|x|^{2}}\right)^{s} d w d s \\
& =\frac{t}{\pi^{\frac{n}{2}}|x|^{n}} H_{1,1 ; 1,0 ; 0,1}^{0,1 ; 0,1 ; 1,0}\left[\begin{array}{c|c}
4 c^{2} t^{2 \alpha}|x|^{-2} & (0 ; 1,1) ;\left(1-\frac{n}{2}, 1\right) ;- \\
a t^{\alpha} & (-1 ; 2 \alpha, \alpha) ;-;(0,1)
\end{array}\right]
\end{aligned}
$$

where the double Mellin-Barnes integral is convergent because the conditions (4) are fulfilled. For $G_{1}^{\alpha}$ we obtain, analogously, under the condition $-\frac{n}{2}<\Re(s)<-\frac{n}{4}$, the following representations:

$$
\begin{aligned}
G_{1}^{\alpha}(x, t)= & \frac{|x|^{1-\frac{n}{2}}}{(2 \pi)^{\frac{n}{2}}} \int_{0}^{+\infty} \tau^{\frac{n}{2}} E_{(\alpha, 2 \alpha), 1}\left(-a t^{\alpha},-c^{2} \tau^{2} t^{2 \alpha}\right) J_{\frac{n}{2}-1}(\tau|x|) d \tau \\
& +\frac{a t^{\alpha}|x|^{1-\frac{n}{2}}}{(2 \pi)^{\frac{n}{2}}} \int_{0}^{+\infty} \tau^{\frac{n}{2}} E_{(\alpha, 2 \alpha), 1+\alpha}\left(-a t^{\alpha},-c^{2} \tau^{2} t^{2 \alpha}\right) J_{\frac{n}{2}-1}(\tau|x|) d \tau \\
= & \frac{1}{\pi^{\frac{n}{2}}|x|^{n}} \frac{1}{(2 \pi i)^{2}} \int_{\mathcal{L}_{1}} \int_{\mathcal{L}_{2}} \frac{\Gamma(1+s+w) \Gamma\left(\frac{n}{2}+s\right) \Gamma(-w)}{\Gamma(1+\alpha w+2 \alpha s)}\left(a t^{\alpha}\right)^{w}\left(\frac{4 c^{2} t^{2 \alpha}}{|x|^{2}}\right)^{s} d w d s \\
& +\frac{a t^{\alpha}}{\pi^{\frac{n}{2}}|x|^{n}} \frac{1}{(2 \pi i)^{2}} \int_{\mathcal{L}_{1}} \int_{\mathcal{L}_{2}} \frac{\Gamma(1+s+w) \Gamma\left(\frac{n}{2}+s\right) \Gamma(-w)}{\Gamma(1+\alpha+\alpha w+2 \alpha s)}\left(a t^{\alpha}\right)^{w}\left(\frac{4 c^{2} t^{2 \alpha}}{|x|^{2}}\right)^{s} d w d s
\end{aligned}
$$




$$
\begin{gathered}
=\frac{1}{\pi^{\frac{n}{2}}|x|^{n}} H_{1,1 ; 1,0 ; 0,1}^{0,1 ; 0,1 ; 1,0}\left[\begin{array}{c|c}
4 c^{2} t^{2 \alpha}|x|^{-2} & (0 ; 1,1) ;\left(1-\frac{n}{2}, 1\right) ;- \\
a t^{\alpha} & (0 ; 2 \alpha, \alpha) ;-;(0,1)
\end{array}\right] \\
+\frac{a t^{\alpha}}{\pi^{\frac{n}{2}}|x|^{n}} H_{1,1 ; 1,0 ; 0,1}^{0,1 ; 0,1 ; 1,0}\left[\begin{array}{c|c}
4 c^{2} t^{2 \alpha}|x|^{-2} & (0 ; 1,1) ;\left(1-\frac{n}{2}, 1\right) ;- \\
a t^{\alpha} & (-\alpha ; 2 \alpha, \alpha) ;-;(0,1)
\end{array}\right] .
\end{gathered}
$$

We end this section solving a fractional Cauchy problem.

Theorem 3 Jlet $x \in \mathbb{R}^{n}, t>0,0<\alpha \leq 1, a \geq 0$, and $c>0$, then the fractional Cauchy problem

$$
\left\{\begin{array}{l}
\left({ }^{C} \partial_{t}^{2 \alpha}+a^{C} \partial_{t}^{\alpha}-c^{2} \Delta_{x}\right) h(x, t)=0 \\
h(x, 0)=f_{1}(x) \\
\frac{\partial h}{\partial t}(x, 0)=f_{2}(x),
\end{array}\right.
$$

is solvable, and its solution has the form

$$
h(x, t)=\int_{\mathbb{R}^{n}} G_{1}^{\alpha}(x-z, t) f_{1}(z) d z+\int_{\mathbb{R}^{n}} G_{2}^{\alpha}(x-z, t) f_{2}(z) d z,
$$

where $G_{1}^{\alpha}$ and $G_{2}^{\alpha}$ are the first and second fundamental solutions given by (28) and (25), respectively, and provided that the integrals in the right-hand side of (29) are convergent.

Remark 3/2 Theorem 3.1, when $a=0$ the solution (29) coincides with the one presented in [12, Cor.6.4] (with $\beta=2 \alpha$ ). Moreover, considering $a=0$ the first fundamental solution $G_{1}^{\alpha}$ coincides with the expression presented in [7] for the fundamental solution of the time-fractional diffusion-wave operator (with $\beta=2 \alpha$ ).

\section{Telegraph process with Brownian time}

Now we consider in (8) $n=1, a=2 \lambda \geq 0, C_{0}=1$ and $C_{1}=0$, i.e., we consider the following time-fractional telegraph equation of order $2 \alpha$

$$
\left({ }^{C} \partial_{t}^{2 \alpha}+2 \lambda^{C} \partial_{t}^{\alpha}-c^{2} \partial_{x x}^{2}\right) u(x, t)=0,
$$

where $x \in \mathbb{R}, t>0, c>0$, and subject to the initial condition $u(x, 0)=\delta(x)$ for $0<\alpha \leq \frac{1}{2}$, while, for $\frac{1}{2}<\alpha \leq 1$, besides the previous condition, also $u_{t}(x, 0)=0$ is assumed. Equation (30) was already studied in [15], where the authors presented only an integral representation for the Fourier transform of the fundamental solution. Moreover, for $\alpha=\frac{1}{2}$ it was obtained an integral representation of the fundamental solution based on the Fourier inversion transform (see Theorem 4.2 in [15]).

Physically, if we consider $\alpha=\frac{1}{2}$ in (30), we obtain a heat equation with damping term which depends on all values of $u$ in $[0, t]$ and assigning an overwhelming weight to those close to $t$ (see [15]). The damping effect of the fractional derivative reverberates on the distribution $u$, where the solution of the heat equation (governing term) is perturbed by the telegraph distribution (which represents the impact of the fractional derivative). Moreover, since the fundamental solution $u$ of (30) reduces, in this particular case, to $G_{1}^{\frac{1}{2}}$ (see (19)), it can be understood as the distribution of a particle moving back and forth the real line with velocities $\pm c$ for a random 
time interval. Making $n=1, a=2 \lambda$ and $\alpha=\frac{1}{2}$ in (26), (27) and (28) we get the following representations $G_{1}^{\frac{1}{2}}$ :

$$
\begin{aligned}
& G_{1}^{\frac{1}{2}}(x, t)=\frac{1}{\pi} \int_{0}^{+\infty} E_{\left(\frac{1}{2}, 1\right), 1}\left(-2 \lambda \sqrt{t},-c^{2} \tau^{2} t\right) \cos (\tau|x|) d \tau \\
& +\frac{2 \lambda \sqrt{t}}{\pi} \int_{0}^{+\infty} E_{\left(\frac{1}{2}, 1\right), \frac{3}{2}}\left(-2 \lambda \sqrt{t},-c^{2} \tau^{2} t\right) \cos (\tau|x|) d \tau \\
& =\frac{1}{\sqrt{\pi}|x|} \frac{1}{(2 \pi i)^{2}} \int_{\mathcal{L}_{1}} \int_{\mathcal{L}_{2}} \frac{\Gamma(1+s+w) \Gamma\left(\frac{1}{2}+s\right) \Gamma(-w)}{\Gamma\left(1+\frac{w}{2}+s\right)}(2 \lambda \sqrt{t})^{w}\left(\frac{4 c^{2} t}{|x|^{2}}\right)^{s} d w d s \\
& +\frac{2 \lambda \sqrt{t}}{\sqrt{\pi}|x|} \frac{1}{(2 \pi i)^{2}} \int_{\mathcal{L}_{1}} \int_{\mathcal{L}_{2}} \frac{\Gamma(1+s+w) \Gamma\left(\frac{1}{2}+s\right) \Gamma(-w)}{\Gamma\left(\frac{3}{2}+\frac{w}{2}+s\right)}(2 \lambda \sqrt{t})^{w}\left(\frac{4 c^{2} t}{|x|^{2}}\right)^{s} d w d s \\
& =\frac{1}{\sqrt{\pi}|x|} H_{1,1 ; 1,0 ; 0,1}^{0,1 ; 0,1 ; 1,0}\left[\begin{array}{c|c}
4 c^{2} t|x|^{-2} & (0 ; 1,1) ;\left(\frac{1}{2}, 1\right) ;- \\
2 \lambda \sqrt{t} & \left(0 ; 1, \frac{1}{2}\right) ;-;(0,1)
\end{array}\right] \\
& +\frac{2 \lambda \sqrt{t}}{\sqrt{\pi}|x|} H_{1,1 ; 1,0 ; 0,1}^{0,1 ; 0,1 ; 1,0}\left[\begin{array}{c|c}
4 c^{2} t|x|^{-2} & (0 ; 1,1) ;\left(\frac{1}{2}, 1\right) ;- \\
2 \lambda \sqrt{t} & \left(-\frac{1}{2} ; 1, \frac{1}{2}\right) ;-;(0,1)
\end{array}\right] \text {. }
\end{aligned}
$$

\section{Acknowledgement}

The work of M. Ferreira, M.M. Rodrigues and N. Vieira was supported by Portuguese funds through the CIDMA - Center for Research and Development in Mathematics and Applications, and the Portuguese Foundation for Science and Technology ("FCT-Fundação para a Ciência e a Tecnologia"), within project UID/MAT/ $0416 / 2013$.

N. Vieira was also supported by FCT via the FCT Researcher Program 2014 (Ref: IF/00271/2014).

\section{References}

[1] R.G. Buschman, H-functions of two variables I, Indian J. Math., 20, (1978), 132-153.

[2] M. Caputo, Linear Models of Dissipation Whose Q Is Almost Frequency Independent-II, Geophys. J. R. Astron. Soc., 13-No.5, (1967), 529-539.

[3] R.F. Camargo, A.O. Chiacchio and E.C. de Oliveira, Differentiation to fractional orders and the fractional telegraph equation, J. Math. Phys., 49-No.3, (2008), Article ID 033505, 12p.

[4] R.C. Cascaval, E.C. Eckstein, L.C. Frota and J.A. Goldstein, Fractional telegraph equations, J. Math. Anal. Appl., 276-No.1, (2002), 145-159.

[5] J. Chen, F. Liu, V. Anh, Analytical solution for the time-fractional telegraph equation by the method of separating variables, J. Math. Anal. Appl., 338-No.2, (2008), 1364-1377.

[6] A. Erdélyi, W. Magnus, F. Oberhettinger and G. Tricomi, Tables of integral transforms-Vol.II, Bateman Manuscript Project, California Institute of Technology, McGraw-Hill Book Company, New York-Toronto-London, 1954.

[7] M. Ferreira and N. Vieira, Fundamental solutions of the time fractional diffusion-wave and parabolic Dirac operators, J. Math. Anal. Appl., 447-No.1, (2017), 329-353.

[8] M. Ferreira, M.M. Rodrigues and N. Vieira, First and second fundamental solutions of the time-fractional telegraph equation with Laplace or Dirac operators, Adv. Appl. Clifford Algebr., 28-No.2, (2018), 28-42.

[9] M. Ferreira, M.M. Rodrigues and N. Vieira, Fundamental solution of the multi-dimensional time fractional telegraph equation, Fract. Calc. Appl. Anal., Fract. Calc. Appl. Anal., 20-No.4, (2017), 868-894. 
[10] M. Ferreira, M.M. Rodrigues and N. Vieira, Fundamental solution of the time-fractional telegraph Dirac operator, Math. Meth. in Appl. Sc., 40-No.18, (2017), 7033-7050.

[11] H.J. Haubold, A.M. Mathai and R.K. Saxena, Mittag-Leffler functions and their applications, J. Appl. Math., (2011), Article ID 298628, $51 \mathrm{p}$.

[12] A. Kilbas, H.M. Srivastava and J.J. Trujillo, Theory and applications of fractional differential equations, NorthHolland Mathematics Studies-Vol.204, Elsevier, Amsterdam, 2006.

[13] Y. Luchko and R. Gorenflo, An operational method for solving fractional differential equations with the Caputo derivatives Acta Math. Vietnam., 24-No.2, (1999), 207-233.

[14] M.O. Mamchuev, Solutions of the main boundary value problems for the time-fractional telegraph equation by the Green function method, Fract. Calc. Appl. Anal., 20-No.1, (2017), 190-211.

[15] E. Orsingher and L. Beghin, Time-fractional telegraph equations and telegraph processes with Brownian time, Probab. Theory Relat. Fields, 128-No.1, (2004), 141-160.

[16] I. Podlubny, Fractional Differential Equations. An introduction to fractional derivatives, fractional differential equations, to methods of their solution and some of their applications, Mathematics in Science and Engineering 198, Academic Press, San Diego, 1999.

[17] S.G. Samko, A.A. Kilbas and O.I. Marichev, Fractional integrals and derivatives: theory and applications, Gordon and Breach, New York, NY, 1993.

[18] R.C. Tautz and I. Lerche, Application of the three-dimensional telegraph equation to cosmic-ray transport, Res. Astron. Astrophys., 16(10) (2016), 162.

[19] S. Yakubovich and M.M. Rodrigues, Fundamental solutions of the fractional two-parameter telegraph equation, Integral Transforms Spec. Funct., 23-No.7, (2012), 509-519. 